\title{
Optimización de la resistencia a compresión usando un diseño de mezcla de vértices extremos, en concretos ternarios basados en residuo de mampostería y cal hidratada
}

\section{Resumen}

Yimmy Fernando Silva URRego ${ }^{1}$

JUAN ERNESTO ROJAS ${ }^{1}$

JEFFRY ALEXANDER GAMBOA ${ }^{1}$

MARISOL GORDILLO ${ }^{2}$

Silvio DelVASTO ${ }^{1}$

La búsqueda de materiales alternativos que sustituyan parcialmente el cemento Portland debido a la problemática ambiental que presenta este en su producción, nace la necesidad de investigar el empleo de mezclas ternarias (cemento Portland de uso general (OPC), residuo de mampostería (RM) y cal hidratada (CAL) para mejorar las propiedades de los concretos y disminuir su impacto ambiental. En este sentido, esta investigación realizo un diseño de mezclas de vértices extremos para estudiar el efecto del empleo de RM proveniente de residuos de construcción y demolición (RCD) y CAL como sustitución parcial del cemento Portland (hasta un 20 \% en peso) en concretos ternarios en la resistencia a la compresión a los 28 días de curado. Por otra parte, se evaluó las propiedades en estado fresco (asentamiento) y endurecido, como la resistencia a la compresión, tracción indirecta, absorción y porosidad y succión capilar. Los resultados mostraron que a mayor porcentaje de reemplazo de RM y menores de CAL presentaron mejores resistencias a la compresión que cuando se presentaba mayores porcentajes de cal hidratada. La resistencia a la compresión aumentó en 25,1\% y $16,1 \%$ a los 28 y 60 días de curado con respecto a la mezcla patrón.

Palabras claves: Concreto ternario, Cemento Portland, Residuo de mampostería, Cal hidratada, Resistencia a la compresión, Residuos de construcción y demolición.

1 Universidad del Valle, Cali, Colombia.

2 Universidad Autónoma de Occidente, Cali, Colombia.

Autor de correspondencia: Silva Urrego, Y.F. (Yimmy Fernando): Universidad del Valle, Facultad de Ingeniería, Escuela de Ingeniería de Materiales: Calle 13 \# 100-00, Edf. 349. Teléfono: +57 (2) 3312251 . Correo electrónico: yimmy.silva@correounivalle.edu.co
Historia del artículo:

Artículo recibido: 13-X-2017/ Aprobado: 18-XII-2018

Disponible online: 3 de febrero de 2019

Discusión abierta hasta octubre de 2020 


\title{
Optimization of Compressive Strength Using Design of Extreme Vertices Mixing, in Ternary Concretes Based on Masonry Residue and Hydrated Lime
}

\begin{abstract}
The search for alternative materials that partially substitute Portland cement is important because of the environmental problems that this presents in its production, hence the need to investigate the use of ternary mixtures (Portland cement for general use (OPC), masonry residue (RM) and hydrated lime (CAL) to improve concrete properties and reduce their environmental impact. According to this, the present research did a design of extreme vertices mixing to study the effect of the use of RM from construction and demolition waste (RCD) and CAL as partial substitution of Portland cement (up to $20 \%$ by weight) in concrete ternary in the compressive strength after 28 days of curing. On the other hand, the properties in fresh state were evaluated (slump) and hardened state, such as compressive strength, indirect tensile strength, absorption and porosity and capillary suction. The results showed that a higher percentage of MR replacement and lower CAL had better compressive strengths than when higher percentages of hydrated lime were present. The compressive strength increased by $25.1 \%$ and $16.1 \%$ at 28 and 60 days of curing with respect to the reference mix.
\end{abstract}

Keywords: Ternary concretes, Portland cement, Masonry residue, Hydrated lime, Compressive strength, Construction and demolition waste.

\section{Otimização da resistência a compressão usando desenho de mescla de vértices estremos, em concretos ternários baseados em resíduos de alvenaria e cal hidratada}

\section{Resumo}

Na busca de materiais alternativos que consigam substituir parcialmente o cimento portland devido ao problema ambiental que gera em sua produção, nasce a necessidade de pesquisar o uso de misturas ternarias (cimento Portland de uso geral (OPC)), resíduo de alvenaria (RM) e cal hidratada (CAL) para melhorar as propriedades dos concretos e diminuir seu impacto ambiental. Neste contexto, a presente pesquisa fez um desenho de vértices extremos misturando para estudar o efeito do uso de RM de resíduos de construção e demolição (RCD) e CAL como substituição parcial do cimento Portland (até 20\% em peso) em ternário de concreto na resistência à compressão após 28 dias de cura. Por outro lado, as propriedades em estado fresco foram avaliadas (slump) e estado endurecido, como resistência à compressão, resistência à tração indireta, absorção e porosidade e sucção capilar. Os resultados mostrarem que maiores porcentagens de substituição de RM e menores de CAL apresentam maiores resistências a compressão que com o aumento no conteúdo de cal hidratada. A resistência aumentos em 21 e $16 \%$ aos 28 e 60 dias de curado respeito a amostra padrão.

Palavras chave: Concreto ternarias, Cimento portland, Resíduo de alvenaria, Cal hidratada, Resistência a compressão, Residuos deconstrução e demolição. 


\section{Introducción}

En la actualidad, uno de los problemas ambientales más profundos y nocivos que circunda al mundo es el calentamiento global y la contaminación. La demanda de los productos orgánicos e inorgánicos ha incrementado esta problemática por el consumismo innecesario de estos, generando residuos sólidos de diferentes fuentes, teniendo algunos un mayor impacto ambiental con respecto a otros. Se estima que en la ciudad de Cali, Colombia, debido a todos los procesos en el campo de la construcción, involucrando procesamiento de materias primas, producción o fabricación de materiales al igual que la demolición de edificaciones e infraestructuras, se generan diariamente un promedio de $2.480 \mathrm{~m}^{3}$ de estos residuos sólidos, de los cuales sólo el 40\% es reutilizado (Ortiz y Silva, 2013). El mal aprovechamiento de estos residuos y los grandes volúmenes de escombros generados, han llevado a que las industrias tengan la necesidad de darles una nueva utilidad para clasificarlos como una familia de materias primas las cuales se pueden valorizar y reutilizar con diferentes fines, entre ellos procesos industriales como la reincorporación de estos residuos en materiales compuestos de matriz cementicia, tal como el concreto o mediante la producción de elementos prefabricados (DAGMA, 2015; Mattey, et al. 2014).

Por otra parte, el concreto y el mortero al ser el material más extensamente usado en la construcción (Pliya \& Cree, 2015), el cual entre sus ingredientes principales se encuentra el cemento Portland (OPC), quien contribuye entre el 5 - 7\% del total de las emisiones antropogénicas globales de $\mathrm{CO}_{2}$, y por lo tanto es un objetivo importante mitigar estas (Fry, 2013; Worrell, et al. 2009; Malhotra, 2000). Consecuentemente un gran reto para la industria de la construcción es satisfacer la necesidad de consumo y al mismo tiempo reducir las emisiones de $\mathrm{CO}_{2}$. Con base a estas razones, varios estudios se han centrado en encontrar alternativas para reemplazar el cemento Portland (Aprianti, 2017), encontrando materiales provenientes de diferentes fuentes, como son los subproductos agroindustriales, industriales o marinos, entre otros. En su mayoría, estos materiales vienen a través de procesos naturales o artificiales (acciones humanas). A nivel mundial se produce una gran cantidad de desechos tales como cenizas volantes, ceniza de cascarilla de arroz, humo de sílice y escoria de alto horno (Yu, et al. 2017; Wang, et al. 2017; Pedro, et al. 2017; Dadsetan y Bai, 2017; Antiohos, et al. 2014). El uso de estos subproductos o desechos contribuye a resolver los problemas de manejo de estos materiales, reduce el costo del concreto y da una solución satisfactoria a los problemas ambientales (Aprianti, 2017).

Sin embargo, hay un desecho o escombro proveniente de los residuos de construcción y demolición (RCD) o por proceso de la industria cerámica que ha sido poco estudiado y sería un gran aporte a la problemática actual que existe en Colombia sobre el manejo de estos desechos. Aproximadamente el 45\% de los RCD se atribuye a productos cerámicos tales como ladrillos, tejas y Porcelana (Reig, et al. 2013), ya que el ladrillo es una de las unidades de mampostería más común empleada en el medio de la construcción. Los ladrillos convencionales se producen a partir de la mezcla de materias primas de arcilla, como material plástico, con la adición de arena, como material no plástico, y se cocen en el horno a una temperatura que varía de 850 a $950{ }^{\circ} \mathrm{C}$. La producción anual mundial de ladrillos se sitúa actualmente en alrededor de 1391 mil millones de unidades y se espera que la demanda de ladrillos esté subiendo continuamente a medida que el proceso de construcción aumente en todo el mundo (Zawrah, et al. 2016; Zhang, 2013). Estos residuos, pueden ser empleados como material cementicio suplementario ya que cuando la arcilla se coce a temperaturas entre 500 y $900{ }^{\circ} \mathrm{C}$, ocurre un proceso de deshidroxilacion en los minerales arcillosos, favoreciendo la formación del metacaolin, el cual es un material amorfo con alto grado de reactividad dando lugar a la actividad puzolánica de la arcilla (capacidad de reacción con hidróxido de calcio y agua para formar compuestos 
cementicios (Schackow et al., 2015). Un estudio sobre el empleo de del residuo de mampostería (RM) como adición a concretos autocompactante se llevó a cabo para analizar su efecto como sustitución del cemento, obteniendo resultados satisfactorios tanto en estado fresco y endurecido con sustituciones del 10 y $20 \%$ en peso del CPO por RM (Silva, et al. 2015).

Por otra parte, el hidróxido de calcio $\left(\mathrm{Ca}(\mathrm{OH})_{2}\right)$, o cal hidratada se utilizó durante siglos en todos los tipos de morteros como un polvo o como una masilla. Se diferencia de los aglutinantes hidráulicos utilizados actualmente (ej: OPC) porque endurece lentamente, debido a una reacción que ocurre entre la cal y el $\mathrm{CO}_{2}$ de la atmósfera, esta reacción genera carbonato de calcio $\left(\mathrm{CaCO}_{3}\right)$. Pero también el $\mathrm{Ca}(\mathrm{OH})_{2}(\mathrm{CH})$ puede combinarse con la sílice $\left(\mathrm{SiO}_{2}\right)$ y la alúmina $\left(\mathrm{Al}_{2} \mathrm{O}_{3}\right)$ presente en los materiales puzolánicos y agua (necesario para la hidratación) para formar silicatos de calcio y aluminatos de calcio (Matias et al., 2014).

El presente trabajo, tiene como objetivo evaluar el empleo del residuo de mampostería y pasta (en masilla) de cal apagada como materiales suplementarios al cemento Portland para la producción de concreto para fines de construcción. Para ello se evaluaron varias mezclas ternarias de OPC, $\mathrm{RM}$ y CH mediante un diseño de mezclas de vértices extremos.

\section{Programa experimental}

\subsection{Materiales}

Cemento Portland de uso general de acuerdo a la NTC 121 (Tipo UG) fue usado para todas las mezclas de concreto. La composición química y las características físicas se muestran en la Tabla 1. El residuo de mampostería fue obtenido a partir de una remodelación de una obra pública (Edificio localizado en la Universidad del Valle) como se aprecia en la Figura 1. Este RCD se adecuó mediante una molienda primaria en una trituradora de mandíbula y posterior molienda en un molino de bolas.
La composición química del RM empleado como material cementicio suplementario se presenta en la Tabla 1. El RM presenta los requerimientos químicos y físicos citados en la ASTM C618 como son la sumatoria de los componentes químicos $\left(\mathrm{SiO}_{2}+\mathrm{Fe}_{2} \mathrm{O}_{3}+\mathrm{Al}_{2} \mathrm{O}_{3}\right)$ y el índice de actividad puzolánica (IAP) (Tabla 2). La cal utilizada fue una cal comercial (Calco de Colombia ${ }^{\circledR}$ ), y sus características se muestran en la Tabla 1. Se empleó arena de rio como agregado fino con un tamaño máximo de 4,75 mm, y grava triturada con un tamaño máximo de agregado de $19 \mathrm{~mm}$ acorde con los estándares de la ASTM C33. El agregado fino presento una gravedad específica y absorción de agua de $2680 \mathrm{~kg} /$ $\mathrm{m}^{3}$ y $1,79 \%$ respectivamente, y el agregado grueso tiene una absorción de agua de $0,92 \%$ y una gravedad especifica de $2870 \mathrm{~kg} / \mathrm{m}^{3}$. Agua potable fue usada para la elaboración de las mezclas y curado de todos los especímenes de los concretos. Jugo de fique se empleó como aditivo plastificante para mantener la trabajabilidad.

TABLA 1. COMPOSICIÓN QUÍMICA Y CARACTERÍSTICAS FÍSICAS DEL CEMENTO (OPC), RESIDUO DE MAMPOSTERÍA (RM) Y CAL HIDRATADA (CAL)

\begin{tabular}{|c|c|c|c|}
\hline Elemento /oxido & OPC (\%) & RM (\%) & CAL (\%) \\
\hline $\mathrm{SiO}_{2}$ & 19,39 & 54,78 & 1,64 \\
\hline $\mathrm{Al}_{2} \mathrm{O}_{3}$ & 4,13 & 21,98 & 0,81 \\
\hline $\mathrm{CaO}$ & 55,66 & 2,62 & 66,83 \\
\hline $\mathrm{Fe}_{2} \mathrm{O}_{3}$ & 4,7 & 9,46 & 0,34 \\
\hline $\mathrm{MgO}$ & 1,71 & 3,76 & 4,46 \\
\hline $\mathrm{Na}_{2} \mathrm{O}$ & 0,32 & 1,43 & - \\
\hline $\mathrm{K}_{2} \mathrm{O}$ & 0,28 & 1,69 & - \\
\hline $\mathrm{SO}_{3}$ & 3,9 & 1,14 & 0,53 \\
\hline P.I. ${ }^{*}$ & 9,21 & 2,51 & 25,14 \\
\hline $\begin{array}{l}\text { Gravedad específica } \\
\qquad\left(\mathrm{kg} / \mathrm{m}^{3}\right)\end{array}$ & 3090 & 2623 & 2480 \\
\hline $\begin{array}{l}\text { Tamaño medio de } \\
\text { partícula }(\mu \mathrm{m})\end{array}$ & 22,70 & 21,69 & 34,80 \\
\hline
\end{tabular}


TABLA 2. PROPIEDADES QUÍMICAS Y FÍSICAS DE PUZOLANAS DE ACUERDO A LA ASTM C618

\begin{tabular}{|c|c|c|}
\hline \multirow[b]{2}{*}{ Requerimiento } & Puzolana & \\
\hline & $\begin{array}{c}\text { clase } N \text { (ASTM } \\
\text { C618) }\end{array}$ & RM \\
\hline \multicolumn{3}{|l|}{$\begin{array}{c}\text { Requerimiento } \\
\text { Químico }\end{array}$} \\
\hline $\begin{array}{c}\mathrm{SiO}_{2}+\mathrm{Al}_{2} \mathrm{O}_{3}+\mathrm{Fe}_{2} \mathrm{O}_{3} \\
(\%)\end{array}$ & Min. 70,0 & 86,22 \\
\hline $\mathrm{SO}_{3}(\%)$ & Max. 4,0 & 1,14 \\
\hline $\begin{array}{l}\text { Contenido de } \\
\text { humedad (\%) }\end{array}$ & Max. 3,0 & \\
\hline $\begin{array}{c}\text { Perdida por ignición } \\
(\%)\end{array}$ & Max 10,0 & 2,51 \\
\hline \multicolumn{3}{|l|}{ Requerimiento Físico } \\
\hline $\begin{array}{c}\text { Cantidad retenida en } \\
\text { tamiz de } 45(\%)\end{array}$ & Max. 34 & \\
\hline $\begin{array}{c}\text { IAP a los } 7 \text { días, } \\
\text { porcentaje de control }\end{array}$ & Min. 75 & - \\
\hline $\begin{array}{l}\text { IAP a los } 28 \text { días, } \\
\text { porcentaje de control }\end{array}$ & Min. 75 & 127,8 \\
\hline
\end{tabular}

\section{Figura 1. Residuo de mampostería empleado}

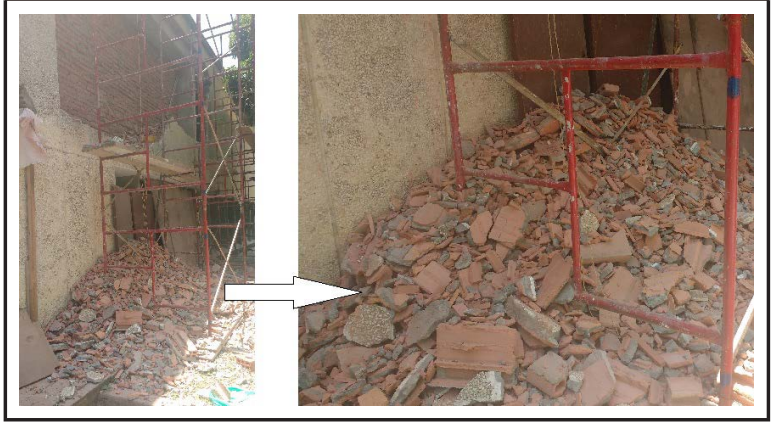

\subsection{Proporciones de Mezcla y Prepa- ración}

El diseño de mezcla del concreto se llevó a cabo para obtener una resistencia de $18 \mathrm{MPa}$ a los 28 días de curado y una trabajabilidad (asentamiento) de $35 \mathrm{~mm}$ - $50 \mathrm{~mm}$ según la American Concrete Institute ACI 211.1. El RM y CAL reemplazaron al OPC de $0 \%$ a $20 \%$ en peso para un contenido total de material cementicio de $350 \mathrm{~kg} / \mathrm{m}^{3}$, la proporción entre los agregados se estableció mediante la curva de Fuller y Thompson (57\% arena y $43 \%$ grava) y la relación agua / aglutinante (a/c) se mantuvo constante en 0,54 mediante el empleo de jugo de fique como aditivo plastificante. Los detalles de las mezclas del concreto se muestran en la Tabla 3. La producción del concreto se realizó en un mezclador (trompo) de 50 litros de capacidad. En primera instancia se introdujeron en el trompo la arena y grava y se realizó un mezclado con un tercio del total de agua de mezcla durante un minuto. La cantidad total de OPC u OPC+RM+CH se mezclaron en un recipiente separado y luego se incorporaron el trompo con otro tercio del agua y se mezcló durante dos minutos. Finalmente se añade el agua restante con la dosis de jugo de fique según sea el caso y se mezcló durante 180 minutos para producir el concreto fresco.

\subsection{Diseño de experimento}

En la Tabla 4 se presentan las condiciones de diseño, con el fin de maximizar la resistencia a la compresión, relacionada con las proporciones de CAL, RM y OPC, se planteó un diseño de mezclas de vértices extremos, el cual implica sólo una porción del espacio de experimentación de un simplex, debido a las restricciones sobre cada una de las componentes para producir una mezcla óptima. El diseño de vértices extremos genero 13 experimentos (3 puntos vértice, 3 puntos de doble combinación, 3 puntos axiales y 4 puntos centrados), asignados al azar a las unidades experimentales (mezcla de concreto), midiendo como resultado la resistencia a la compresión.

Para el análisis de los resultados, se planteó un modelo cuadrático Sheffe, el cual es representado en la Ecuación 1. El modelo está dado por:

$$
y=\sum_{i=1}^{q} \beta_{i} X_{i}+\sum_{i=1}^{q-1} \sum_{j=i+1}^{q} \beta_{i j} X_{i} X_{j}
$$

Donde la variable de respuesta (y) es la resistencia a la compresión, $\beta$, son los coeficientes lineales del modelo y las Xs representa las componentes de la mezcla para $\mathrm{q}=3$ componentes de la mezcla. Este modelo cuadrático permite involucrar no solo efectos lineales sino también los efectos cuadráticos e interacciones en el caso de ser significativas. 


\begin{tabular}{|c|c|c|c|c|c|c|}
\hline Mezcla & $\begin{array}{c}\text { OPC } \\
\left(\mathrm{kg} / \mathrm{m}^{3}\right)\end{array}$ & $\begin{array}{c}\text { CAL } \\
\left(\mathrm{kg} / \mathrm{m}^{3}\right)\end{array}$ & $\begin{array}{c}\text { RM } \\
\left(\mathrm{kg} / \mathrm{m}^{3}\right)\end{array}$ & $\begin{array}{c}\text { Agua } \\
\left(\mathbf{k g} / \mathrm{m}^{3}\right)\end{array}$ & $\begin{array}{l}\text { Grava } \\
\left(\mathbf{k g} / \mathbf{m}^{3}\right)\end{array}$ & $\begin{array}{l}\text { Arena } \\
\left(\mathrm{kg} / \mathrm{m}^{3}\right)\end{array}$ \\
\hline M1 & 350 & 0 & 0 & 189 & 860 & 1037 \\
\hline M2 & 315 & 0 & 35 & 189 & 860 & 1037 \\
\hline M3 & 280 & 0 & 70 & 189 & 860 & 1037 \\
\hline M4 & 326 & 12 & 12 & 189 & 860 & 1037 \\
\hline M5 & 305 & 23 & 23 & 189 & 860 & 1037 \\
\hline M6 & 292 & 12 & 47 & 189 & 860 & 1037 \\
\hline M7 & 292 & 47 & 12 & 189 & 860 & 1037 \\
\hline M8 & 280 & 35 & 35 & 189 & 860 & 1037 \\
\hline M9 & 315 & 35 & 0 & 189 & 860 & 1037 \\
\hline M10 & 280 & 70 & 0 & 189 & 860 & 1037 \\
\hline
\end{tabular}

\begin{tabular}{|c|c|c|c|c|c|}
\hline Nomenclatura & Orden estadístico & $\begin{array}{c}\text { Número de } \\
\text { mezclas }\end{array}$ & CAL:X1 & $\mathrm{RM}: \mathrm{X} 2$ & OPC:X3 \\
\hline M4 & 8 & 1 & 0,03 & 0,03 & 0,93 \\
\hline M5 & 11 & 2 & 0,07 & 0,07 & 0,87 \\
\hline M1 & 1 & 3 & 0,00 & 0,00 & 1,00 \\
\hline M5 & 7 & 4 & 0,07 & 0,07 & 0,87 \\
\hline M7 & 9 & 5 & 0,13 & 0,03 & 0,83 \\
\hline M5 & 12 & 6 & 0,07 & 0,07 & 0,87 \\
\hline M10 & 2 & 7 & 0,20 & 0,00 & 0,80 \\
\hline M3 & 3 & 8 & 0,00 & 0,20 & 0,80 \\
\hline M8 & 6 & 9 & 0,10 & 0,10 & 0,80 \\
\hline M9 & 5 & 10 & 0,10 & 0,00 & 0,90 \\
\hline $\mathrm{M} 2$ & 4 & 11 & 0,00 & 0,10 & 0,90 \\
\hline M5 & 13 & 12 & 0,07 & 0,07 & 0,87 \\
\hline M6 & 10 & 13 & 0,03 & 0,13 & 0,83 \\
\hline
\end{tabular}

En el caso de este estudio se presentaron tres componentes de la mezcla: CAL, RM y OPC. Con el fin de simplificar la visualización de este diseño, se proporciona una gráfica de diseño simplex de las componentes consideradas en la Figura 2, como puede apreciarse están restringidas y coincide con un triángulo dentro de la región simplex.

\subsection{Métodos de prueba}

\subsubsection{Resistencia a la compresión y trac-} ción indirecta

Cilindros de 7,62 cm de diámetro x 15,24 cm de altura fueron moldeados para la resistencia a la 
compresión y tracción indirecta. Estos ensayos se realizaron en una maquina ELE Soiltest con capacidad de $1000 \mathrm{kN}$ a una velocidad constante. La resistencia a la compresión se determinó a las edades de 7, 28 y 60 curado según la ASTM C39. El ensayo de tracción indirecta se realizó basado en la ASTM C496 a 28 y 60 días de curado. Para cada edad, se ensayaron 3 especímenes y el valor medio de estas es el reportado.

\subsubsection{Propiedades de permeabilidad}

Los ensayos de transporte seleccionados fueron la prueba de absorción y porosidad para determinar la porosidad total y la absorción de acuerdo con la ASTM C642, donde las muestras se secan, sumergen y luego se hierven en agua para determinar el volumen de poros después de 28 y 60 días, y la sorptividad capilar (succión capilar) fue también evaluada mediante la aplicación del procedimiento estándar EMPA-SIA 162/1 (Fagerlund, 1982), en donde se permite que el agua pase a una muestra seca a través de un proceso más gradual de succión capilar, y la masa de la muestra es monitoreada como función del tiempo.

\section{Resultados y discusiones}

\subsection{Resistencia a la compresión}

La resistencia a la compresión de las mezclas de concreto con relación a/c constante se muestran en la Figura 3. Se puede observar que las mezclas con mayor porcentaje de RM (M2, M3, M5 y M6), presentaron las mayores resistencias a largas edades, esto puede ser atribuido a su comportamiento puzolánico, aunque a los 7 días de curado las mezclas con diferentes porcentajes de RM presentaron una menor resistencia a la compresión en comparación a la mezcla de referencia (M1), como el caso de las mezcla M2 y M3 que solo tenían adición de RM y presentaron una disminución cercana a 13\% y 5,8\% respectivamente. Sin embargo, la mezcla M5 presento una mayor resistencia en un 4,87\% y la mezcla M6 en un 9,64\% a los 7 días de curado. A mayores edades se observa el efecto beneficioso en la resistencia de las mezclas que presentan en su composición el RM. A los 60 días la ganancia de resistencia de la mezcla M6 fue de 13,22\% con respecto a M1, lo que apunta que a mayores edades de curado el efecto puzolánico de esta adición sería más notoria (Schackow, et al. 2015).

Figura 2. Diseño simplex restringido en proporciones de las componentes

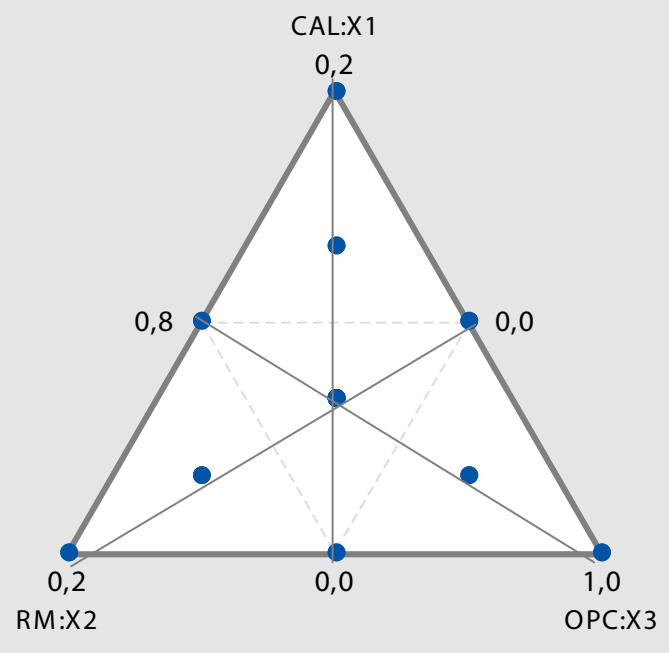




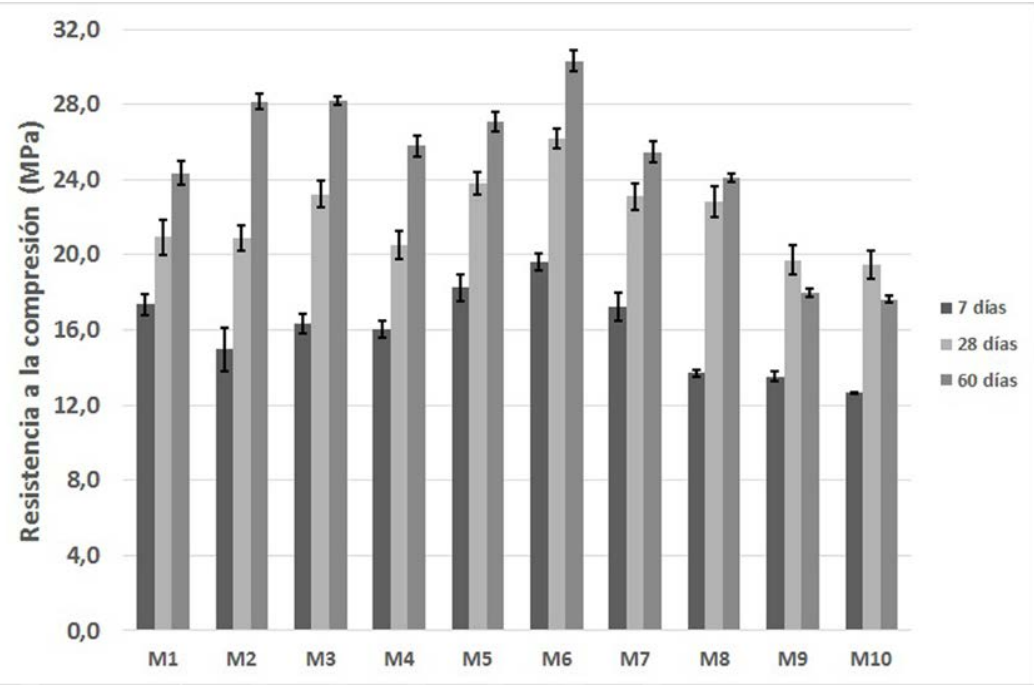

\subsubsection{Análisis de diseño de experi- mentos}

La resistencia de las muestras a los 28 días de curado oscilaron entre 16 y 26,21 MPa, La mayoría de las mezclas presentaron valores por encima del objetivo propuesto de $18 \mathrm{MPa}$ para las aplicaciones básicas de construcción, a excepción de los concretos que contenían $20 \%$ de CAL y $80 \%$ de OPC como cementante los cuales presentaron una resistencia de $16 \mathrm{MPa}$. Con el fin de medir estos efectos, en la Tabla 5, se muestra el análisis de varianza, se ajustó un modelo cuadrático utilizando la técnica de mínimos cuadrados.
En la Tabla 5, se muestra el análisis de varianza para determinar la significancia de la regresión, el cual cumple con los supuestos sobre el error del modelo a niveles de significancia mayores del 10\%. Inicialmente se encontró que el modelo planteado es adecuado a un nivel de significancia del 65,6\% (p-valor) y con respecto a las interacciones entre dos efectos no fueron significativos, sin embargo la interacción de tercer orden si es significativa (p-valor 0,045), en el modelo se dejó la interacción de segundo orden CAL*RM dado que mejora el ajuste del modelo con un $\mathrm{R}^{2}$ de $83,6 \%$ considerado un valor alto, indicando que estos términos contribuyen a la explicación de la resistencia a la compresión presentada por el concreto.

TABLA 5. ANÁLISIS DE VARIANZA DE LA RESISTENCIA A LA COMPRESIÓN $\left(R^{2}=83.6 \%\right)$

\begin{tabular}{c|c|c|c|c|c|} 
Fuente & GL & Suma de cuadrados & Cuadrados medios & F & P-valor \\
\hline Regresión & 4 & 98,489 & 24,622 & 10,19 & 0,003 \\
\hline Lineal & 2 & 63,491 & 31,626 & 13,09 & 0,003 \\
\hline CAL:X1*RM:X2 & 1 & 21,414 & 5,838 & 2,42 & 0,159 \\
\hline CAL:X1*RM:X2*OPC:X3 & 1 & 13,583 & 13,583 & 5,62 & 0,045 \\
\hline Error residual & 8 & 19,322 & 2,415 & & \\
\hline Falta de ajuste & 5 & 10,49 & 2,098 & 0,71 & 0,655 \\
\hline Error puro & 3 & 8,832 & 2,944 & & \\
\hline Total & 12 & 117,811 & & & \\
\hline
\end{tabular}


La ecuación que se puede utilizar para optimizar la resistencia a la compresión está dada en la

\section{Ecuación 2.}

Resistencia a la compresión $=15,24 \mathrm{CAL}+$

$24,4 \mathrm{RM}+20,39 \mathrm{OPC}+11,46 \mathrm{CAL} * \mathrm{RM}+$

$71,98 \mathrm{CAL}^{*} \mathrm{RM} * \mathrm{OPC}$

\section{Optimización de la resistencia a la compresión}

A partir de la Ecuación 2, se planteó la optimización (maximizar) de la variable de respuesta (resistencia a la compresión), para un valor mínimo de $18 \mathrm{MPa}$ con un valor objetivo de $23 \mathrm{MPa}$. En la Figura 4, se muestra una alta predicibilidad $(D=1)$, para valores de resistencia a la compresión de 24 $\mathrm{MPa}$, cuando se tiene condiciones como:
Cal: 5,49\%, RM: 14,51\%, OPC: $80 \%$

Cal: 0,99\%, RM: 19,01\%, OPC: 80\%

Los valores de CAL es posible que usufructúen entre 0 y 5,3\% aproximadamente y RM entre 16 y $20 \%$, mientras se le adicione el mínimo de cemento (80\%) y la predicción de la resistencia se mantiene en aproximadamente $24 \mathrm{MPa}$.

En la Figura 5, se presenta el gráfico de contornos de la resistencia a la compresión, en este se evidencia que efectivamente para valores de OPC cercanos al $80 \%$ con valores de RM cercanos al 20\% y valores bajos de CAL se obtienen resistencias a la compresión de aproximadamente $24 \mathrm{MPa}$, es decir el área de las resistencias más alta se encuentra en el vértice izquierdo de la gráfica.

\section{Figura 4. Optimización de la Resistencia a la compresión}

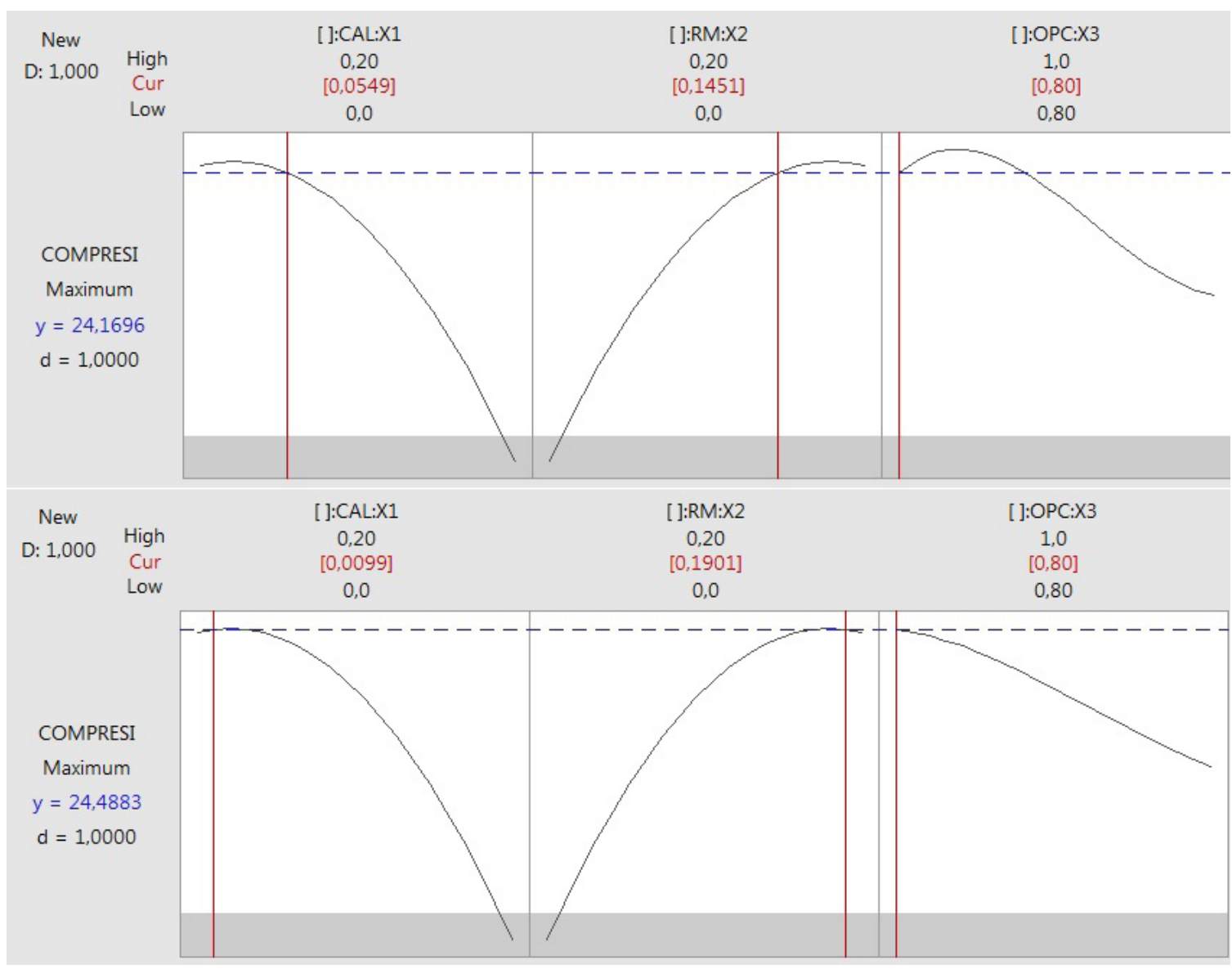




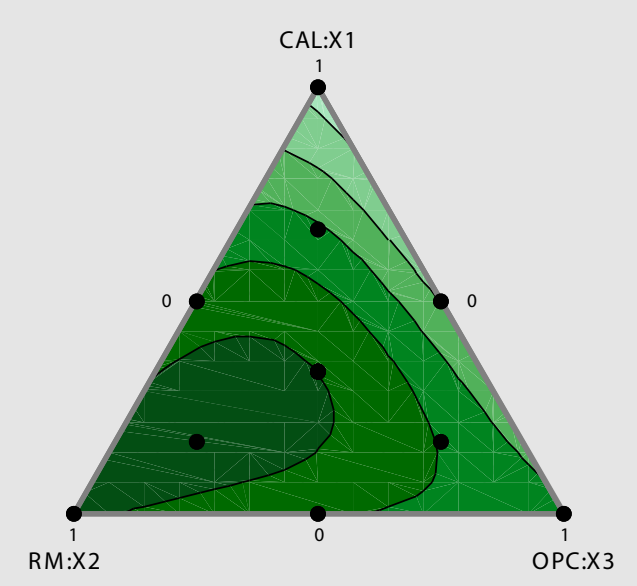

Figura 6. Resistencia a la tracción indirecta de las mezclas con adiciones de Residuo de Mampostería y Cal hidratada como reemplazo del Cemento Portland

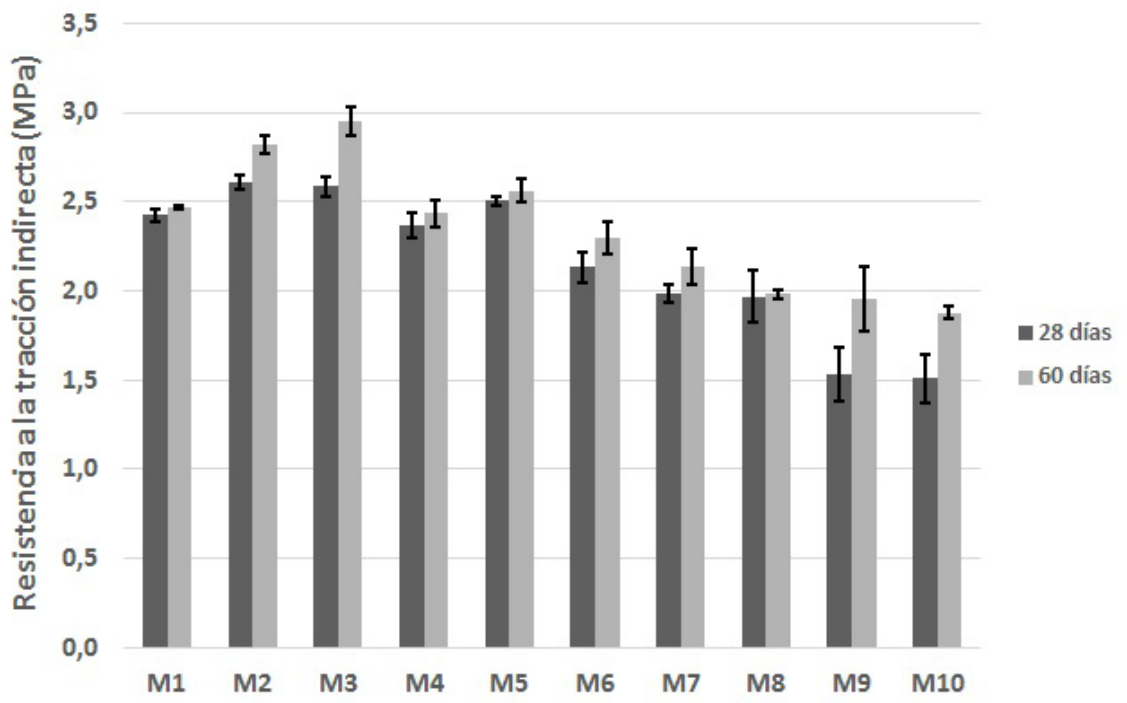

\subsection{Resistencia a la tracción indirecta}

La resistencia a la tracción indirecta se muestra en la Figura 6, en donde se puede observar la misma tendencia mostrada en los concretos evaluados a la resistencia a la compresión, debido a que estos dos ensayos guardan una relación entre sí. La resistencia a la tracción indirecta depende de varios factores como el tipo de agregado o la distribución de tamaño de partícula. Se puede observar que las mezclas con mayores cantidades de residuo de mampostería (M2 y M3) comparada con M1 (mezcla de referencia) y las demás mezclas, mostraron mayores resistencia a la tracción indirecta, este comportamiento puede ser atribuido a la forma y la estructura del residuo de mampostería ya que al tener una alta rugosidad, tiene una mayor fuerza adhesiva 
con las partículas de cemento (Ge, et al. 2015). Al momento de aumentar el porcentaje de cal hidratada en la mezcla, disminuye incluso para las mezclas que contiene residuo de mampostería, esto sucede debido a que la fuerza adhesiva entre las partículas de cal hidratada, residuo de mampostería y cemento es menor. Al aumentar la cal hidratada, hay un mayor número de partículas de este material, y por ende una menor presencia de RM y CPO dentro de la mezcla lo que hace que disminuya la resistencia.

\subsection{Prueba de permeabilidad del agua}

\subsubsection{Absorción, densidad y porosidad}

El porcentaje de absorción, densidad y porosidad de las mezclas planteadas en el diseño de experimentos se determinaron mediante las directrices de la ASTM C642 y los resultados se registraron en la Tabla 5 y 6 para 28 y 60 días de curado respectivamente.

TABLA 5. RESULTADOS DE ABSORCIÓN, DENSIDAD Y POROSIDAD DE LAS MEZCLAS A 28 DÍAS DE CURADO

\begin{tabular}{c|c|c|c|c|c|} 
PROBETA & $\begin{array}{c}\text { \% Absorción } \\
\text { Después de } \\
\text { Inmersión }\end{array}$ & $\begin{array}{c}\text { Densidad de } \\
\text { Masa Seca }\end{array}$ & $\begin{array}{c}\text { Densidad de } \\
\text { Masa Después de } \\
\text { Inmersión }\end{array}$ & $\begin{array}{c}\text { Densidad } \\
\text { Aparente }\end{array}$ & $\begin{array}{c}\text { \% Volumen } \\
\text { de poros } \\
\text { Permeables }\end{array}$ \\
\hline M1 & 7,153 & 2,077 & 2,218 & 2,452 & 15,270 \\
\hline M2 & 6,414 & 2,048 & 2,178 & 2,403 & 14,743 \\
\hline M3 & 7,300 & 2,216 & 2,369 & 2,656 & 16,572 \\
\hline M4 & 6,904 & 2,069 & 2,207 & 2,423 & 16,252 \\
\hline M5 & 6,147 & 2,214 & 2,356 & 2,607 & 15,559 \\
\hline M6 & 6,327 & 2,253 & 2,392 & 2,648 & 14,876 \\
\hline M7 & 7,341 & 2,120 & 2,268 & 2,510 & 15,078 \\
\hline M9 & 6,853 & 2,217 & 2,365 & 2,648 & 14,620 \\
\hline
\end{tabular}

TABLA 6. RESULTADOS DE ABSORCIÓN, DENSIDAD Y POROSIDAD DE LAS MEZCLAS A 60 DÍAS DE CURADO

\begin{tabular}{c|c|c|c|c|c} 
PROBETA & $\begin{array}{c}\text { \% Absorción } \\
\text { Después de } \\
\text { Inmersión }\end{array}$ & $\begin{array}{c}\text { Densidad de } \\
\text { Masa Seca }\end{array}$ & $\begin{array}{c}\text { Densidad de } \\
\text { Masa Después de } \\
\text { Inmersión }\end{array}$ & $\begin{array}{c}\text { Densidad } \\
\text { Aparente }\end{array}$ & $\begin{array}{c}\text { \% Volumen } \\
\text { de poros } \\
\text { Permeables }\end{array}$ \\
\hline M1 & 6,135 & 2,372 & 2,518 & 2,800 & 15,261 \\
\hline M2 & 5,807 & 2,312 & 2,446 & 2,708 & 14,652 \\
\hline M3 & 5,987 & 2,314 & 2,453 & 2,700 & 14,289 \\
\hline M4 & 5,893 & 2,233 & 2,364 & 2,622 & 14,086 \\
\hline M5 & 5,332 & 2,321 & 2,445 & 2,666 & 14,676 \\
\hline M6 & 6,025 & 2,340 & 2,480 & 2,733 & 14,392 \\
\hline M7 & 6,229 & 2,291 & 2,433 & 2,685 & 12,902 \\
\hline M8 & 5,792 & 2,324 & 2,459 & 2,705 & 14,861 \\
\hline M10 & 6,046 & 2,242 & 2,377 & 2,612 & 14,176 \\
\hline
\end{tabular}


Se encontró que la mezcla con mayor absorción a los 28 días de curado fue la M10 y la menor M5, con un porcentaje de absorción de 7,469\% y $6,147 \%$ respectivamente, lo que representa un 4,4\% más de absorción en la mezcla M10 y 4,4\% menos de absorción en la mezcla M5 en comparación con la mezcla de referencia (M1). A los 60 días de curado todas las mezclas presentaron una menor absorción y volumen de poros permeables. La menor absorción de las mezclas con presencia de RM en su composición con respecto a la mezcla M1 es posiblemente debido al refinamiento de poros (Ge, et al. 2015; Schackow, et al. 2015). Con respecto al aumento de la cal hidratada, a los 28 y 60 días de curado se observa que tiene un mayor porcentaje de absorción, menor densidad y mayor volumen de poros permeables ya que a excesivas cantidades de cal hidratada o $\mathrm{Ca}(\mathrm{OH})_{2}$, que también se presenta en la reacción de hidratación de cemento como un subproducto y más conocido como portlandita, se considera excesivo su presencia en la mezcla, por lo que esta precipita en el medio y actúa como un agente expansivo, que ayuda a aumentar la porosidad del concreto (Damene, et al. 2016).

Con respecto al porcentaje del volumen de poros permeables, en la Figura 7 se aprecia el comportamiento de este a los 28 y 60 días de curado.

En la Figura 7 se observa el comportamiento de las mezclas con respecto al porcentaje de poros permeables, en donde las mezclas ternarias con cemento, cal hidratada y residuo de mampostería (M4, M5, M6, M7 y M8) reduce la porosidad de las mezclas, debido al efecto puzolánico que tiene el RM y el hidróxido de calcio adicional que hay en la mezcla, lo que genera una reacción puzolánica resultando en la formación de un gel llamado tobermorita que contiene silicatos/aluminatos cálcicos hidratados, el cual es el mismo producto que se genera en la hidratación del cemento. Este gel reduce el volumen de los poros permeables, por lo tanto aumenta la densidad y disminuye la absorción (Schackow, et al. 2015; Dopico, et al. 2008).

Figura 7. Resistencia a la compresión de las mezclas con adiciones de Residuo de Mampostería y Cal hidratada como reemplazo del Cemento Portland

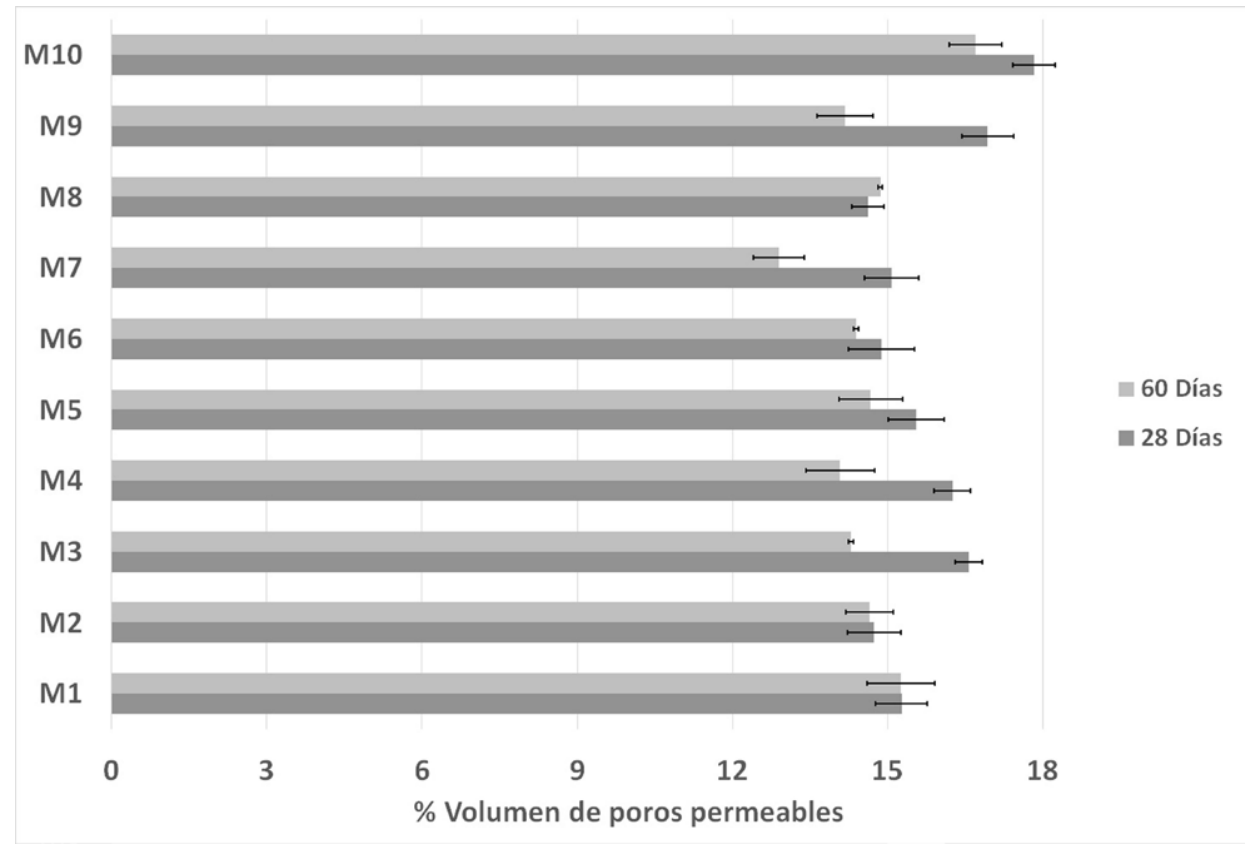




\subsubsection{Succión Capilar}

Los resultados de succión capilar de las mezclas diseñadas experimentalmente se muestran en las Tablas 7 y 8 a los 28 y 60 días respectivamente.

TABLA 7. RESULTADOS DE SUCCIÓN CAPILAR DE LAS MEZCLAS A 28 DÍAS DE CURADO

\begin{tabular}{|c|c|c|c|}
\hline Mezcla & $\mathrm{K}\left(\mathrm{Kg} / \mathrm{m}^{2} \mathrm{seg}^{1 / 2}\right)$ & $\mathrm{m}\left(10^{7} \mathrm{~s} / \mathrm{m}^{2}\right)$ & $\begin{array}{l}\text { \% Porosidad } \\
\text { efectiva ( } \varepsilon e)\end{array}$ \\
\hline M1 & 0,0277 & 4,995 & 19,489 \\
\hline M2 & 0,0272 & 2,204 & 12,787 \\
\hline M3 & 0,0200 & 3,760 & 11,986 \\
\hline M4 & 0,0239 & 2,399 & 11,603 \\
\hline M5 & 0,0238 & 2,512 & 11,628 \\
\hline M6 & 0,0271 & 2,124 & 12,514 \\
\hline M7 & 0,0354 & 1,703 & 14,601 \\
\hline M8 & 0,0236 & 2,658 & 12,033 \\
\hline M9 & 0,0236 & 3,367 & 13,608 \\
\hline M10 & 0,0273 & 3,199 & 15,451 \\
\hline
\end{tabular}

A los 28 días de curado, la rata de absorción primaria se ve favorecida con la incorporación de la cal hidratada en la dosificación de las diferentes mezclas, consiguiendo el valor más alto de todas las mezclas. La mezcla M7 (87\% CPO, 7\% CH y 7\% RM) presenta el mayor coeficiente de absorción capilar (K) $0,0354 \mathrm{Kg} / \mathrm{m}^{2} \mathrm{seg}^{1 / 2}$ y el menor valor de la resistencia a la penetración de agua 1,703 x 107 s/m²; esto afecta a la durabilidad del concreto.

TABLA 8. RESULTADOS DE SUCCIÓN CAPILAR DE LAS MEZCLAS A 60 DÍAS DE CURADO

\begin{tabular}{|c|c|c|c|}
\hline Mezcla & $\mathrm{K}\left(\mathrm{Kg} / \mathrm{m}^{2} \mathrm{seg}^{1 / 2}\right)$ & $m\left(10^{7} s / m^{2}\right)$ & $\begin{array}{l}\% \text { Porosidad } \\
\text { efectiva ( } \varepsilon e \text { ) }\end{array}$ \\
\hline M1 & 0,0219 & 3,615 & 13,134 \\
\hline M2 & 0,0247 & 2,736 & 12,931 \\
\hline M3 & 0,0243 & 3,258 & 13,848 \\
\hline M4 & 0,0251 & 3,121 & 13,814 \\
\hline M5 & 0,0244 & 3,104 & 13,547 \\
\hline M6 & 0,0231 & 3,279 & 13,197 \\
\hline M7 & 0,0226 & 3,679 & 13,583 \\
\hline M8 & 0,0229 & 3,433 & 13,208 \\
\hline M9 & 0,0232 & 3,525 & 13,748 \\
\hline M10 & 0,0261 & 3,307 & 14,592 \\
\hline
\end{tabular}

A los 60 días de curado, se aprecia que en la mayoría de los casos, la tendencia de la resistencia a la penetración de agua para muestras es aumentar, esto puede ser debido a una mayor hidratación del cemento y la reacción puzolánica de las mezclas con RM. Las mezclas con mayor resistencia a la penetración (m) (M4, M5, M6, M7 y M8) tienden a tener una mejor durabilidad en el concreto debido a que al tener un mayor m, es más complicado la penetración de agentes externos en el concreto, además que al hidratarse toda la mezcla (Cemento, puzolana y cal hidratada), generan cristales que bloquean los poros y esto hace este concreto menos permeables (Barbhuiya, et al. 2009). Cabe recordar que la reacción que produce el residuo de mampostería y la cal hidratada se forman a edades tardías de curado, característico de las reacciones puzolánicas (Matias, et al. 2014).

\section{Conclusiones}

En el presente estudio, se investigó sistemáticamente las propiedades en estado endurecido de las mezclas ternarias de $\mathrm{CPO}, \mathrm{RM}$ y $\mathrm{CH}$ en concreto. Con base en los resultados, se pueden extraer las siguientes conclusiones:

El empleo del residuo de mampostería proveniente de los residuos de construcción y demolición en mezcla con cal hidratada son una alternativa viable como reemplazo del cemento hasta en un $20 \%$. Ya que de esta forma se da un aprovechamiento a los RM, los cuales son una problemática ambiental debido a su alto volumen producido en remodelaciones, construcciones y demoliciones.

Cuando la cantidad de residuo de mamposteria es mayor en la mezcla, la resistencia a la compresión presenta un mejor desempeño en comparación al patrón, cuando la mezcla presenta mayores cantidades de cal hidratada.

La sinergia observada en las mezclas ternarias $\mathrm{CPO} / \mathrm{RM} / \mathrm{CH}$ se atribuye a los efectos físicos y químicos, como son el efecto filler del $\mathrm{CH}$ y puzolánico del RM. 


\section{Agradecimientos}

Los autores desean expresar su agradecimiento a: Grupo de materiales Compuestos (GMC) de la Escuela de Ingeniería de Materiales de la Universidad del Valle y Centro de Excelencia de Nuevos Materiales (CENM); al proyecto FP44842-399- 2015: "Investigación de un material cementicio ecoeficiente para elementos de construcción de bajo costo" financiado por COLCIENCIAS- "El Patrimonio Autónomo Fondo Nacional de Financiamiento para la Ciencia, la Tecnología y la Innovación, Francisco José de Caldas y al Departamento Administrativo de Ciencia y Tecnología (Colciencias)".

\section{Referencias}

Antiohos, S.K.; Papadakis, V.G.; Tsimas, S. (2014). Rice husk ash (RHA) effectiveness in cement and concrete as a function of reactive silica and fineness. Cement and Concrete Research, 61-62, pp. 20-27. [Online] Disponible en: https://doi.org/10.1016/j. cemconres.2014.04.001. [Consultado 23 de agosto de 2017].

Aprianti, (2017). A huge number of artificial waste material can be supplementary cementitious material (SCM) for concrete production e a review part II. Journal of Cleaner Production, 142, pp. 4178-4194. [Online] Disponible en: https://doi.org/10.1016/j. jclepro.2015.12.115. [Consultado 23 de agosto de 2017].

Barbhuiya, S.A.; Gbagbo J.K.; Russell, M.I.; Basheer P.A.M. (2009). Properties of fly ash concrete modified with hydrated lime and silica fume. Construction and Building Materials, 23, pp. 3233-3239. [Online] Disponible en: https://doi:10.1016/j.conbuildmat.2009.06.001. [Consultado 25 de agosto de 2017].

Dadsetan, S., Bai, J. (2017). Mechanical and microstructural properties of self-compacting concrete belnded with metakaolin, ground granulated blast-furnace slag and fly ash. Construction and Building Materials, 146, pp. 658-667. [Online] Disponible en: https:// doi.org/10.1016/j.conbuildmat.2017.04.158. [Consultado 23 de agosto de 2017].
DAGMA. (2015). Plan de gestión integral de residuos sólidos de Santiago de Cali. Santiago de Cali: Alcaldia de Santiago de Cali.

Damene, Z.; Goual, M.S.; Houessou, J.; Dheilly, R.M.; Goullieux, A.; Quéneudec, M. (2016). The use of southern Algeria dune sand in cellular lightweight concrete manufacturing: effect of lime and aluminium content on porosity, compressive strength and thermal conductivity of elaborated materials. European Journal of Environmental and Civil Engineering 8189 (April 2017), pp. 1-17. [Online] Disponible en: http://dx.doi.org/10.1080/19648189.2016.12562 33. [Consultado 25 de agosto de 2017].

Dopico, J.J.; Hernandez, F.M.; Day, R.L.; Middendorf, B.; Gehrke, M.; Martinez, M. (2008). Desarrollo de hormigones con aglomerantes cal-puzolana fina como material cementicio suplementario. Revista Ingeniería de Construcción, 23, pp. 171-178. [Online] Disponible en: http://dx.doi.org/10.4067/S071850732008000300005. [Consultado 23 de agosto de 2017].

Fagerlund, G. (1982). On the capillarity of concrete, Nordic Concrete Research 1, 6.1-6.20.

Fry, M. (2013). Cement, carbon dioxide, and the 'necessity' narrative: A case study of Mexico. Geoforum, 49, pp. 127-138. . [Online] Disponible en: https://doi. org/10.1016/j.geoforum.2013.06.003. [Consultado 23 de julio de 2017].

Ge, Z.; Wang, Y.; Sun, R.; Wu, X.; Guan, Y. (2015). Influence of ground waste clay brick on properties of fresh and hardened concrete. Construction and Building Materials 98, pp. 128-136. [Online] Disponible en: https://doi.org/10.1016/j.conbuildmat.2015.08.100. [Consultado 25 de julio de 2017].

Malhotra, V.M. (2000). Role of supplementary cementing materials in reducing greenhouse gas emissions. In: Gjorv, O.E., Sakai, K. (Eds.), Concrete technology for a Sustainable Development in the 21st Century. E\&FN Spon, London, 226-235.

Matias, G.; Faria, P.; Torres, I. (2014). Lime mortars with heat treated clays and ceramic waste: A review. Construction and Building Materials, 73, pp. 125-136. [Online] Disponible en: https://doi.org/10.1016/j. conbuildmat.2014.09.028. [Consultado 25 de agosto de 2017].

Mattey, P.; Robayo, R.; Silva Y.; Alvarez, N.; Delvasto, S. (2014). Caracterización física y mecánica de 
agregados reciclados obtenidos a partir de escombros de la construcción. Informador técnico, 78 (2) pp. 121-127. [Online] Disponible en: https://doi. org/10.23850/22565035.95. [Consultado 25 de agosto de 2017].

Ortiz, H.; Silva, M.S. (2013). ¿De dónde vienen y a donde van a parar los escombros de Cali?. El PAIS. Disponible en: http://www.elpais.com.co/elpais/ graficos/infografia-ruta-escombros-cali.

Pedro D.; de Brito J.; Evangelista L. (2017). Evaluation of high-performance concrete with recycled aggregates: use of densified silica fume as cement replacement. Construction and Building Materials, 147, pp. 803-814. [Online] Disponible en: https:// doi.org/10.1016/j.conbuildmat.2017.05.007. [Consultado 25 de agosto de 2017].

Pliya, P.; Cree, D. (2015). Limestone derived eggshell powder as a replacement in Portland cement mortar. Construction and Building Materials, 95, pp. 1-9. [Online] Disponible en: https://doi.org/10.1016/j. conbuildmat.2015.07.103. [Consultado 25 de agosto de 2017].

Reig, L.; Tashima, M.; Borrachero, M.; Monzó, J.; Cheeseman, C.; Payá, J. (2013). Properties and microstructure of alkali-activated red clay brick waste. Construction and Building Materials, 43, pp. 98-106. [Online] Disponible en: https://doi.org/10.1016/j. conbuildmat.2013.01.031. [Consultado 25 de agosto de 2017].

Schackow, A.; Stringari, D.; Senff, L.; Correia, S.L.; Segadães, A.M. (2015). Influence of fired clay brick waste additions on the durability of mortars. Cement \& Concrete Composites, 62, pp. 82-89. [Online] Disponible en: https://doi.org/10.1016/j.cemconcomp.2015.04.019. [Consultado 24 de agosto de 2017].

Silva, Y.; Robayo, R.; Mattey P.; Delvasto, S. (2015). Obtención de concretos autocompactantes empleando residuos de demolición. Rev. LatinAm. Metal. Mat. 35(1), pp. 86-94. [Online] Disponible en: http:// www.rlmm.org/ojs/index.php/rlmm/article/ view/549. [Consultado 24 de agosto de 2017].

Wang, D.; Zhou X.; Meng Y.; Chen, Z. (2017). Durability of concrete containing fly ash and silica fume against combined freezing-thawing and sulfate attack. Construction and Building Materials, 147, pp. 398-406. [Online] Disponible en: https://doi.org/10.1016/j. conbuildmat.2017.04.172. [Consultado 24 de agosto de 2017].

Worrell, E.; Bernstein, L.; Roy, J.; Price, L.; Harnisch, J., (2009). Industrial energy efficiency and climate change mitigation. Energy efficiency, 2, pp. 109123. [Online] Disponible en: https://DOI 10.1007/ s12053-008-9032-8. [Consultado 25 de agosto de 2017].

Yu, J.; Lu, C.; Leung C.K.Y.; Li, G. (2017). Mechanical properties of green structural concrete with ultrahighvolume fly ash. Construction and building materials, 147, pp. 510-518. [Online] Disponible en: https:// doi.org/10.1016/j.conbuildmat.2017.04.188. [Consultado 25 de agosto de 2017].

Zawrah, M.F.; Gado R.A.; Feltin, N.; Ducourtieuxb, S.; Devoille, S. (2016). Recycling and utilization assessment of waste fired clay bricks (Grog) with granulated blast-furnace slag for geopolymer production. Process Safety and Environmental Protection, 103, pp. 237-251. [Online] Disponible en: https://doi. org/10.1016/j.psep.2016.08.001. [Consultado 25 de agosto de 2017].

Zhang, L. (2013). Production of bricks from waste materials - a review, Construction and Building Materials, 47, pp. 643-655. [Online] Disponible en: https:// doi.org/10.1016/j.conbuildmat.2013.05.043. [Consultado 24 de agosto de 2017].

\section{PARA CITAR ESTE ARTÍCULO / TO REFERENCE THIS ARTICLE / PARA CITAR ESTE ARTIGO /}

Silva Urrego, Y.F.; Rojas, J.E.; Gamboa, J.A.; Gordillo, M.; Delvasto, S. (2019). Optimización de la resistencia a compresión usando un diseño de mezcla de vértices extremos, en concretos ternarios basados en residuo de mampostería y cal hidratada. Revista EIA, 16(31), enero-junio, pp. 99-113. [Online]. Disponible en: https://doi.org/10.24050/reia.v16i31.1177 